\title{
An Estimate of Global Anthrax Prevalence in Livestock: A Meta-analysis
}

\author{
Bylaiah Sushma ${ }^{1}$ (D) Seema Shedole ${ }^{1}$ (D), Kuralayanapalya Puttahonnappa Suresh² (D), Gowda Leena ${ }^{3}$ (D), \\ Sharanagouda S. Patil ${ }^{4}$ (D) and Gowda Srikantha²
}

1. Department of Computer Science and Engineering, M S Ramaiah Institute of Technology, Matthikere, Bengaluru, Karnataka, India; 2. Spatial Epidemiology Laboratory, Indian Council of Agricultural Research (ICAR) - National Institute of Veterinary Epidemiology and Disease Informatics (NIVEDI), Yelahanka, Bengaluru, Karnataka, India; 3. Department of Veterinary Public Health and Epidemiology, Veterinary College, Hebbal, Bengaluru, Karnataka, India; 4. Virology

Laboratory, Indian Council of Agricultural Research (ICAR) - National Institute of Veterinary Epidemiology and Disease Informatics (NIVEDI), Yelahanka, Bengaluru, Karnataka, India.

Corresponding author: Bylaiah Sushma, e-mail: sushma.b3@gmail.com

Co-authors: SS: seemas@msrit.edu, KPS: sureshkp97@rediffmail.com, GL: drleenagowda@gmail.com, SSP: sharanspin13@gmail.com, GS: srikanthagowda098@gmail.com

Received: 11-12-2020, Accepted: 06-04-2021, Published online: 22-05-2021

doi: www.doi.org/10.14202/vetworld.2021.1263-1271 How to cite this article: Sushma B, Shedole S, Suresh KP, Leena G, Patil SS, Srikantha G (2021) An Estimate of Global Anthrax Prevalence in Livestock: A Meta-analysis, Veterinary World, 14(5): 1263-1271.

\begin{abstract}
Background and Aim: Anthrax, caused by the soil-borne spore-forming bacteria called Bacillus anthracis, is a zoonotic disease that persists worldwide in livestock and wildlife and infects humans. It is a great hazard to livestock; henceforth, evaluating the global concerns about the disease occurrence in livestock is essential. This study was conducted to estimate the global prevalence of anthrax and predict high-risk regions, which could be an input to veterinarians to take necessary steps to control and avoid the disease.
\end{abstract}

Materials and Methods: A literature review was performed using online databases, namely, PubMed, Google Scholar, Scopus, Biomed Central, and Science Direct, to extract relevant publications worldwide between 1992 and 2020 . Initially, 174 articles were selected, and after scrutinizing, 24 articles reporting the prevalence of anthrax were found to be adequate for the final meta-analysis. The statistical study was accompanied by employing fixed effects and random effects models using R.

Results: The pooled prevalence of anthrax globally was 28\% (95\% confidence interval, 26-30\%) from 2452 samples through the fixed effects model. Continent-wise subgroup analysis through the random effects model revealed that the pooled prevalence of anthrax was highest in Africa (29\%) and least in North America (21\%).

Conclusion: In these publications, anthrax causes economic loss to farmers and, thus, to the world. Hence, controlling anthrax infections in high-risk regions are essential by implementing appropriate control measures to decrease the effect of the disease, thereby reducing economic loss.

Keywords: anthrax, livestock, meta-analysis, prevalence, subgroup analysis.

\section{Introduction}

Anthrax is an ancient and severe disease that causes the loss of livestock. Besides, human beings commonly get infected through contact with infected animals and their products or through working exposure in clinics and agricultural fields. Anthrax is a bacterial infection caused by Bacillus anthracis, an aerobic, Gram-positive spore-forming bacterium and occurs primarily as a cutaneous, pulmonary, or gastrointestinal infection, depending on the route of entry of $B$. anthracis spores [1]. B. anthracis mostly affects grazing animals, namely, cattle, sheep, and goats that can be infested by consuming spores existing in contaminated soils. Gnawing flies might be associated

Copyright: Sushma, et al. Open Access. This article is distributed under the terms of the Creative Commons Attribution 4.0 International License (http://creativecommons.org/licenses/ by/4.0/), which permits unrestricted use, distribution, and reproduction in any medium, provided you give appropriate credit to the original author(s) and the source, provide a link to the Creative Commons license, and indicate if changes were made. The Creative Commons Public Domain Dedication waiver (http:// creativecommons.org/publicdomain/zero/1.0/) applies to the data made available in this article, unless otherwise stated. with disease transmission in certain areas [2]. The spore form of $B$. anthracis in the soil turns inactive and continues for a long time; such that, years may pass between outbreaks. Natural conditions contribute to its transmission, such as higher soil type, calcium levels, increased temperature and humidity, slightly alkaline $\mathrm{pH}$, and higher levels of decaying organic matter, along with the organism's ability to live in a harsh environment for extended survival of the spore in the soil. Moreover, the spore is resistant to sunlight, heat, drying, and many disinfectants [3]. Initially, researchers have suggested that these factors influence vegetative anthrax bacilli. Nonetheless, a study has demonstrated that the vegetative cells of $B$. anthracis have certain supplemental and physiological necessities and are improbable to survive outside the host. A survey of the properties of $B$. anthracis spores and different Bacillus species has recommended that the particular soil factors connected to pandemic regions reflect significant ecological conditions that guide B. anthracis spores in causing ailments. Particularly, significant levels of calcium in the soil may assist with 
keeping up spore essentialness for delayed periods, along with the opportunity of spores experiencing and contaminating another host [1]. When the bacterium invades living organisms, it germinates and starts replicating in the freshly infected animal. Henceforth, control actions are vital to the survival of the livestock populace. The top and globally accepted cost-effective control measures of infection in livestock are annual vaccination and active surveillance program to recognize outbreaks early in the epidemic progression. Besides, the proper disposal of carcasses is crucial for the prolonged endurance of the agent in the soil. The corpses of infected animals should be predisposed without opening to avoid sporulation and further contamination of the soil [3].

Anthrax is global in its geographical distribution and is endemic to many parts of South Europe, Asia, Africa, North and South America, and Australia [1]. This study analyzes the worldwide prevalence of anthrax using a meta-analysis. Metaanalysis is a broadly extending field of research and a formal, quantitative, epidemiological examination used to deliberately survey explorative publications to determine decisions about a huge volume of information. Meta-analyses that are well-guided may act as an important tool for increasing animal productivity and prosperity. Arranging disclosures from various examinations are needed to ensure that meta-analytic exploration is appealing. A huge measure of publication discoveries has been made in animal prosperity and production, making meta-analytic studies more promising [4]. The meta-analysis steps include defining the domain of research and hypothesis; defining consideration/avoidance rules; searching for historical data; choosing the finalized publications; extricating information on factors of interest, coding systems, figuring impact sizes, and understandings; choosing expected moderators; analyzing their relationships; and writing report and critical assessment results [5]. Subgroup analysis includes parting all participant information into subgroups to examine the relationships between them. Subgroup analysis might be accomplished for subsets of publications, for example, different geological areas. Subgroup analysis could be conducted as a method for exploring diverse outcomes.

This study was conducted to estimate the global prevalence of anthrax and predict high-risk regions, which could be an input to veterinarians to take necessary steps to control and avoid the disease.

\section{Materials and Methods}

\section{Ethical approval}

Ethical approval was not necessary as we have not collected any animal samples for the study.

\section{Literature search}

A methodical investigation was performed on the literature about the worldwide prevalence of anthrax in livestock. The information was pooled from the following databases: PubMed, Google Scholars,
Science Direct, Biomed Central, and Scopus. Studies reporting the prevalence of anthrax were systematically reviewed and included for meta-analysis. More than a thousand articles were looked at, assessed, and chosen, and the outcomes were exposed to meta-analysis to decide the prevalence rate of anthrax concerning different periods and different continents. The literature search from various publications was embraced for the period from 1992 to 2020 . Given a huge quantity of literature, we assembled and consolidated the attributes of the publications, such as author, publication year, continent, number of samples tested, number of positive samples, and animals (i.e. species, such as cattle, sheep, goats, and buffalo). The recovery language was restricted to English. Original articles, peer-reviewed articles, and references cited from the retrieved articles were examined again to follow the previous year's publications. The preferred reporting items for systematic review and meta-analysis protocol (http://www.prisma-statement.org) were followed in conducting the study.

\section{Study selection and data extraction}

The publications were confined to studies on the prevalence of anthrax in cows, sheep, goats, and buffalo species worldwide. The gathered articles were completely inspected for replications and were eliminated. The additional standards to consider for meta-analysis are the number of animals tested, number of animals infected, time of study, and publications that have used the standardized confirmatory test, for example, blood smear examination, staining techniques, sero-analysis by enzyme-linked immunosorbent assay (ELISA), and nucleic acid-based methods by polymerase chain reactions (PCRs). The publications referring to outbreak assessments, survey articles, case reports, and clinical primers were dismissed from the examination. Relevant studies were taken in light of the aforementioned standards, and the outcomes from the discrete examinations were pulled out freely to a predesigned data collection sheet. Information extricated from the chosen publications were as follows: The study year, sample size, number of animals positive for $B$. anthracis, method used for diagnosis, author's name, study location, and publication year. The estimation of the prevalence of anthrax for individual studies was intended to choose the uppermost prevalence when various diagnostic methodologies were employed. The stages included for efficient data filtering were as follows: (i) The analysis method used was lucid with the goals of the meta-analysis, and its goals directed a few qualities to be estimated and stated as reports. (ii) Guaranteeing that a chosen study does not have an anomaly concerning quality and relations are essential.

\section{Meta-analysis}

Meta-analysis for publications on prevalence was performed to generate a weighted average ratio of the prevalence of anthrax in numerous publications, 
allowing us to acquire a more precise measure of anthrax prevalence from several publications, consequently giving a superior heading toward imminent work [6]. Precise reviews were used to incorporate discoveries from accessible research publications at the highest quality level to estimate the viability of preventive and restorative mediations for the predetermined settings. The meta-analysis of anthrax prevalence in livestock was conducted using $\mathrm{R}$ (version 3.4.3; R Foundation for Statistical Computing, Vienna, Austria; https://www.R-project.org/). The packages of $\mathrm{R}$ used for meta-analysis were as follows: metafor, meta, and qdap. Forest plots were used to visually represent the meta-analysis. Forest plots show the impact gauge and confidence intervals (CIs) of each examination. Every study is depicted as a square at a point estimate of impact, and a line depicted horizontally broadening both sides of the square portrays a $95 \%$ CI. The square area corresponds to the weight assigned to that review in the meta-analysis. The impact of the model was picked relying on the level of heterogeneity $\left(I^{2}\right)$. The most extreme probability assessor was used to decide between-study variances $\tau^{2}$. Since generous heterogeneity was normal, fixed effects and random-effects models were used to reveal a pooled prevalence of anthrax.

\section{Publication bias}

The chances of publication bias were measured using a funnel plot with the Y-axis representing the standard error of each study and the $\mathrm{X}$-axis representing the Arcsine transformation of the proportion of each study. If the publication bias is nil, high-accuracy investigations lie along the line of normal, whereas low-accuracy investigations scatter equitably on both margins of the normal line, making a funnel-shaped distribution [7]. The dispersion of publications in the funnel formation directs to publication bias. Furthermore, funnel plot deflection was curbed using the rank correlation strategy, Egger's test, and the linear regression test. The p-value of every null hypothesis test was either rejected or accepted. The Trim and Fill strategy was used to regulate the funnel plot deviation. To decide the level of discrepancy over multiple publications due to heterogeneousness as opposed to risk, the Cochran Q test (Chi-square test of heterogeneity) and heterogeneity statistic $I^{2}$ (Higgins $I^{2}$ ) were determined. To measure the heterogeneity, $I^{2}$ estimations of $25 \%, 50 \%$, and $75 \%$ were used to denote low, medium, and high heterogeneity, respectively [8]. The $\mathrm{H}$ value was computed to encapsulate the effect of heterogeneity. Subsequently, the H statistic, without maximum boundary, will permit fluctuations in heterogeneity with great validness when the quantities of publications are fewer.

\section{Subgroup analysis}

Subgroup analysis is the procedure where all participant data incorporated in the meta-analysis are divided into subgroups, according to trial characteristics, namely, geographical location, or patient characteristics, namely, gender, and then a meta-analysis is performed on one or more of these subsets. Sources of heterogeneity can be found by conducting a subgroup analysis [9]. In this study, subgroup analysis was conducted to assess the heterogeneity among publications from different continents. The impact of the model was selected relying on the level of heterogeneity $\left(I^{2}\right)$. The most extreme probability assessor was used to decide betweenstudy variances $\tau^{2}$. A random-effects model was used to show a pooled prevalence of anthrax from a global perspective.

\section{Statistical analysis}

A two-stage methodology was adopted in the statistical aspects of a meta-analysis. The summary statistics from each analysis were determined in the first level. The summary statistics from each sample were combined in the second level to provide an aggregate result. We performed the first level with the help of Rayyan systematic review and Zotero software. Second level was conducted by employing fixed effects and random-effects meta-analysis. A fixed-effect meta-analysis assumes that all observed variance was due to chance, i.e., sampling error within the sample. The random-effects model, on the other hand, allows for differences between experiments. Since the random vs. fixed-effect model is generally the appropriate model, meta-analysis was conducted using fixed effect and random effects model and subgroup analysis was conducted by employing random effects model in the present study to estimate global anthrax prevalence.

\section{Results}

\section{Details of prevalence publications}

The online database searches returned 2875 likely articles related to the keyword search. Review publications on the prevalence of anthrax in humans were omitted. After the initial scrutiny of the titles of eligible articles, those that have reported the prevalence of anthrax were selected, whereas those that were irrelevant were excluded from the study. Subsequently, 174 articles were retained after the initial assessment. Among them, after analyzing the abstracts, 94 were removed, and a further 42 articles were removed by subsequently analyzing the full article. Thirty-eight publications were selected, and after the final data scrutiny, 24 articles were included in the meta-analysis. The addition and omission benchmarks followed for the meta-analysis of the studies on the prevalence of anthrax are presented in Figure-1.

\section{Meta-analysis of anthrax prevalence in livestock}

This study covered five continents: Africa, Asia, North America, Europe, and Australia. No suitable publications were available for South America. The number of publications included in the meta-analysis was 24, including 2452 samples, from 1992 to 2020. 
The meta-analysis designated that inconsistency was more among the publications $\left(\tau^{2}=0.0162\right.$; heterogeneity $I^{2}=87 \%$; heterogeneity variability $H^{2}=7.11$; $\mathrm{p}<0.01)$. The pooled prevalence by the fixed effects models was $28 \%$ (95\% CI, 26-30\%). The pooled prevalence by the random-effects model was $24 \%(95 \%$ CI, 20-29\%) (Figure-2) [10-33].
Asymmetry of the funnel plots was found using the Begg and Mazumdar rank correlation test, with the results showing that Kendall's tau was 0.08 with a $\mathrm{p}=0.61$, and the Eggers regression test using a fixed-effects meta-regression model, with the results showing that $z$ was -5.28 with $\mathrm{p}<0.01$. The results demonstrated generous deviation in the funnel plots

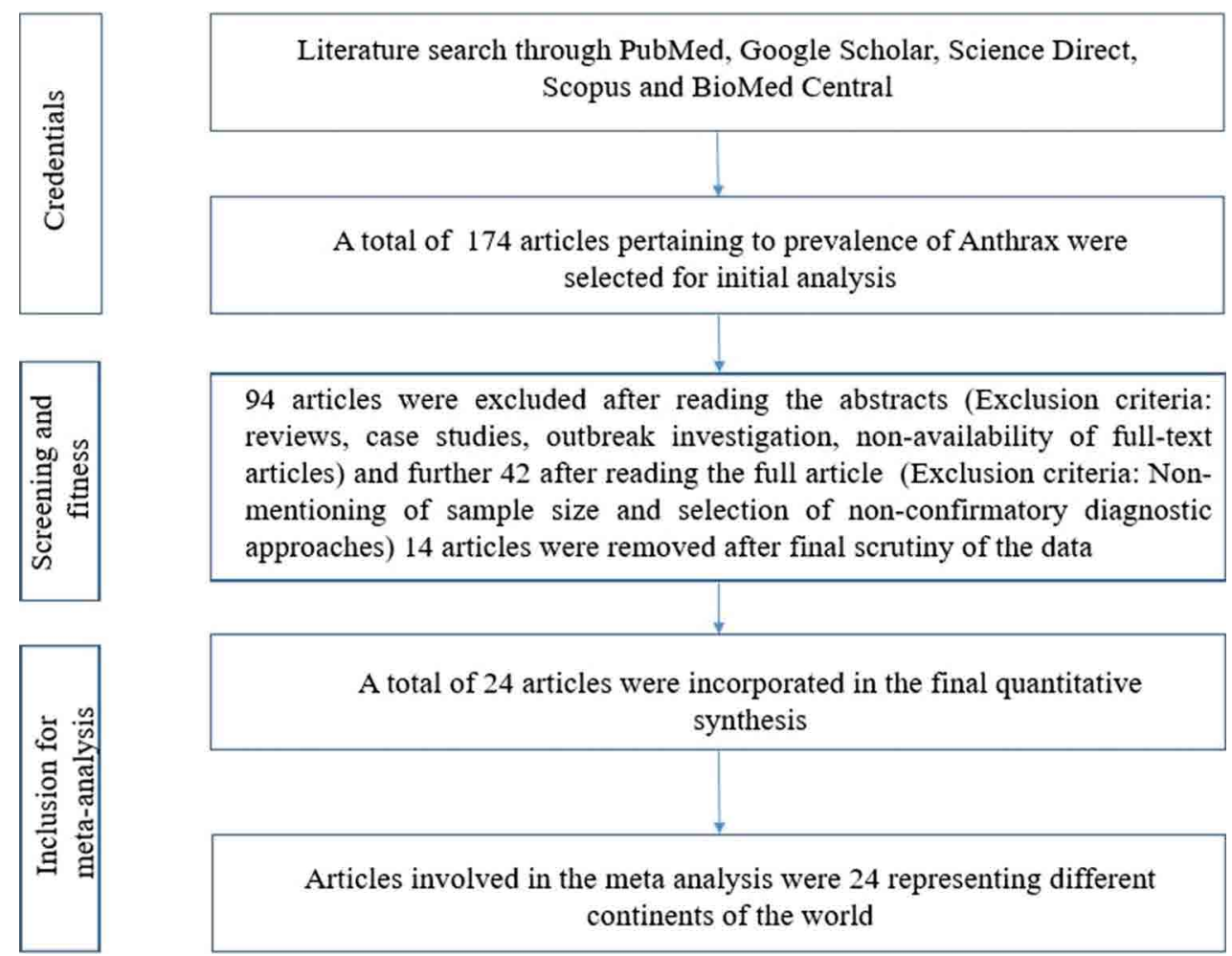

Figure-1: Schematic diagram showing the literature search with exclusion/inclusion procedure for meta-analysis.

\begin{tabular}{|c|c|c|c|c|c|c|c|}
\hline Study & \multicolumn{2}{|c|}{ Events Total } & & \multirow{2}{*}{$\begin{array}{r}\text { Proportion } \\
0.38\end{array}$} & \multirow{2}{*}{$\begin{array}{r}95 \%-\mathrm{Cl} \\
{[0.21 ; 0.56]}\end{array}$} & $\begin{array}{l}\text { Weight } \\
\text { (fixed) }\end{array}$ & $\begin{array}{l}\text { Weight } \\
\text { (random) }\end{array}$ \\
\hline [10] Blackburn et al., 2014_Texas_North America & 12 & 32 & & & & $1.3 \%$ & $3.6 \%$ \\
\hline [11] Chakraborty et al.,2012_Banḡladesh_Asia & 7 & 35 & & 0.20 & {$[0.08 ; 0.37]$} & $1.4 \%$ & $3.7 \%$ \\
\hline [12] Derzelle et al., 2015_Denmark_Europe & 7 & 41 & $\vdots$ & 0.17 & {$[0.07 ; 0.32]$} & $1.7 \%$ & $3.8 \%$ \\
\hline [13] Driciru et al., 2020_Western Uganda_Africa & 11 & 44 & & 0.25 & {$[0.13 ; 0.40]$} & $1.8 \%$ & $3.9 \%$ \\
\hline [14] Ert et al., 2007_Arizona_North America & 32 & 88 & & 0.36 & {$[0.26 ; 0.47]$} & $3.6 \%$ & $4.5 \%$ \\
\hline [15] Gierczynski et al., 2009_Poland_Europe & 6 & 15 & & 0.40 & {$[0.16 ; 0.68]$} & $0.6 \%$ & $2.6 \%$ \\
\hline [16] Irenge et al.,2010_Belgium_Europe & 9 & 50 & & 0.18 & {$[0.09 ; 0.31]$} & $2.0 \%$ & $4.0 \%$ \\
\hline [17] Kamboyi et al.,2019_Zambia_Africa & 33 & 80 & & 0.41 & {$[0.30 ; 0.53]$} & $3.3 \%$ & $4.4 \%$ \\
\hline [18] Kenefic et al.,2008_NorthAmerica & 6 & 47 & & 0.13 & {$[0.05 ; 0.26]$} & $1.9 \%$ & $4.0 \%$ \\
\hline [19] Kolton et al., 2019_Atlanta_USA & 1 & $51+$ & - & 0.02 & {$[0.00 ; 0.10]$} & $2.1 \%$ & $4.0 \%$ \\
\hline [20] Lewerin et al., 2010_Sweden_Europe & 12 & 90 & - & 0.13 & {$[0.07 ; 0.22]$} & $3.7 \%$ & $4.5 \%$ \\
\hline [21] Luna et al., 2005_Florida_USA & 7 & 60 & & 0.12 & {$[0.05 ; 0.23]$} & $2.4 \%$ & $4.2 \%$ \\
\hline [22] Maksimovic et al., 2017_Bosnia and Herzegovina_Europe & 8 & 18 & & 0.44 & {$[0.22 ; 0.69]$} & $0.7 \%$ & $2.8 \%$ \\
\hline [23] Mongoh et al.,2008_NorthDakota_North America & 243 & 576 & + & 0.42 & {$[0.38 ; 0.46]$} & $23.5 \%$ & $5.1 \%$ \\
\hline [24] Muller et al.,2015_Bundoora_Australia & 9 & 58 & & 0.16 & {$[0.07 ; 0.27]$} & $2.4 \%$ & $4.2 \%$ \\
\hline [25] Muturi et al., 2018_Kenya_Africa & 20 & 54 & & 0.37 & {$[0.24 ; 0.51]$} & $2.2 \%$ & $4.1 \%$ \\
\hline [26] Mwakapeje et al., 2019_Tanzania_Africa & 44 & 108 & & 0.41 & {$[0.31 ; 0.51]$} & $4.4 \%$ & $4.6 \%$ \\
\hline [27] Rao et al., 2019_Georgia_Asia & 36 & 138 & & 0.26 & {$[0.19 ; 0.34]$} & $5.6 \%$ & $4.7 \%$ \\
\hline [28] Rondinone et al., 2020_Itāly_Europe & 55 & 234 & 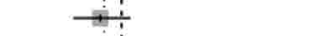 & 0.24 & {$[0.18 ; 0.29]$} & $9.5 \%$ & $4.9 \%$ \\
\hline [29] Ryu et al., 2005_Korea_Asia & 9 & 34 & & 0.26 & {$[0.13 ; 0.44]$} & $1.4 \%$ & $3.6 \%$ \\
\hline [30] Shiferaw et al., 2004_Ethiopia_Africa & 13 & 82 & ؛ & 0.16 & {$[0.09 ; 0.26]$} & $3.3 \%$ & $4.4 \%$ \\
\hline [31] Simbotwe et al., 2018_Zambia_Africa & 23 & 131 & $-\vdots$ & 0.18 & {$[0.11 ; 0.25]$} & $5.3 \%$ & $4.7 \%$ \\
\hline [32] Thierry et al., 2014_France_Europe & 35 & 130 & $\because$ & 0.27 & {$[0.20 ; 0.35]$} & $5.3 \%$ & $4.7 \%$ \\
\hline [33] Turnbull et al.,1992_United Kingdom_Europe & 64 & 256 & $\stackrel{\vdots}{i}$ & 0.25 & {$[0.20 ; 0.31]$} & $10.4 \%$ & $5.0 \%$ \\
\hline Fixed effect model & & 2452 & $\infty$ & 0.28 & {$[0.26 ; 0.30]$} & $100.0 \%$ & - \\
\hline Random effects model & & & $\sum_{1}$ & 0.24 & {$[0.20 ; 0.29]$} & - & $100.0 \%$ \\
\hline Heterogeneity: $I^{2}=87 \%, \tau^{2}=0.0162, p<0.01$ & & & $010000-1$ & & & & \\
\hline & & & $\begin{array}{lllllll}0.1 & 0.2 & 0.3 & 0.4 & 0.5 & 0 & \\
\end{array}$ & & & & \\
\hline
\end{tabular}

Figure-2: Forest plot showing the results of meta-analysis. 
(rejected the null hypothesis), hence uncovering the probable occurrence of publication bias (Figure-3). Inconsistency, heterogeneity, and publication bias

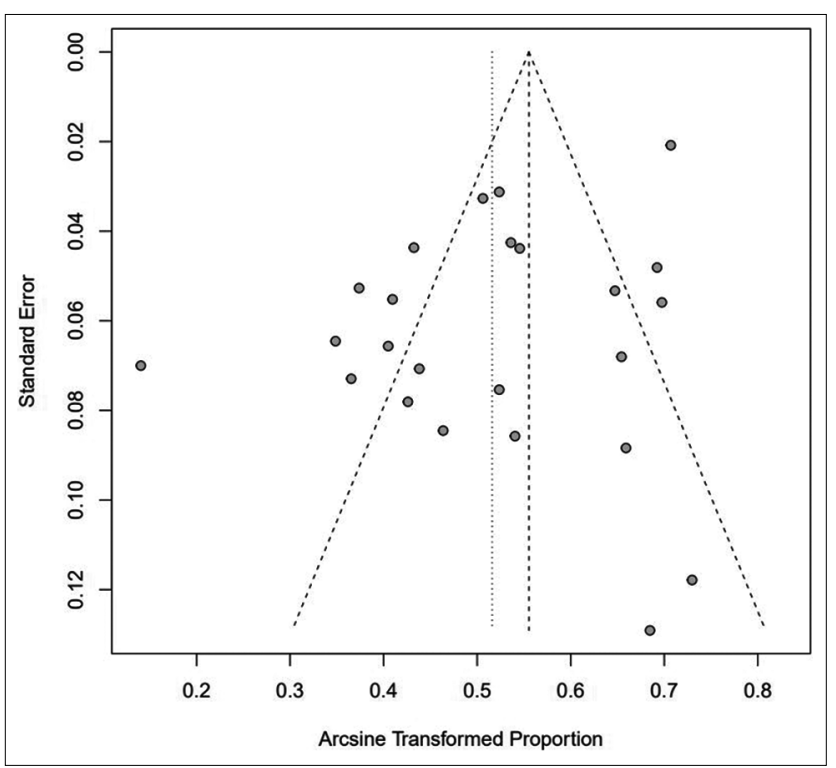

Figure-3: Funnel plot showing publication bias. were observed among the included publications due to which further subgroup analysis was conducted.

\section{Subgroup meta-analysis}

A continent wise study showed that the pooled prevalence of anthrax for the period from 1992 to 2020 in Africa was $29 \%$ (95\% CI, $20-39 \% ; I^{2}=84 \%$; $\left.\tau^{2}<0.0147 ; \mathrm{p}<0.01\right)$, followed by Asia $(25 \% ; 95 \% \mathrm{CI}$, $\left.19-31 \% ; I^{2}=0 \% ; \tau^{2}=0 ; \mathrm{p}=0.73\right)$, Europe $(23 \% ; 95 \%$ CI, $\left.19-28 \% ; I^{2}=53 \% ; \tau^{2}=0.0027 ; \mathrm{p}=0.04\right)$, and North America $\left(21 \%\right.$; 95\% CI, 9-38\%; $I^{2}=95 \% ; \tau^{2} 0.0477$; $\mathrm{p}<0.01)$. Nevertheless, the subgroup analysis showed an overall prevalence of $25 \%(95 \%$ CI, $20-30 \%$; $\left.I^{2}=87 \% ; \tau^{2}=0.0166 ; \mathrm{p}<0.01\right)$, and the residual heterogeneity $I^{2}$ was $87 \%(\mathrm{p}<0.01)$ by the random effects model (Figure-4) [10-33]. The world map of continent wise pooled prevalence of anthrax in livestock based on the results of the subgroup meta-analysis is shown in Figure-5.

\section{Discussion}

Anthrax is a globally distributed disease and is endemic to many parts of Africa, Asia, South Europe, North and South America, and Australia, which

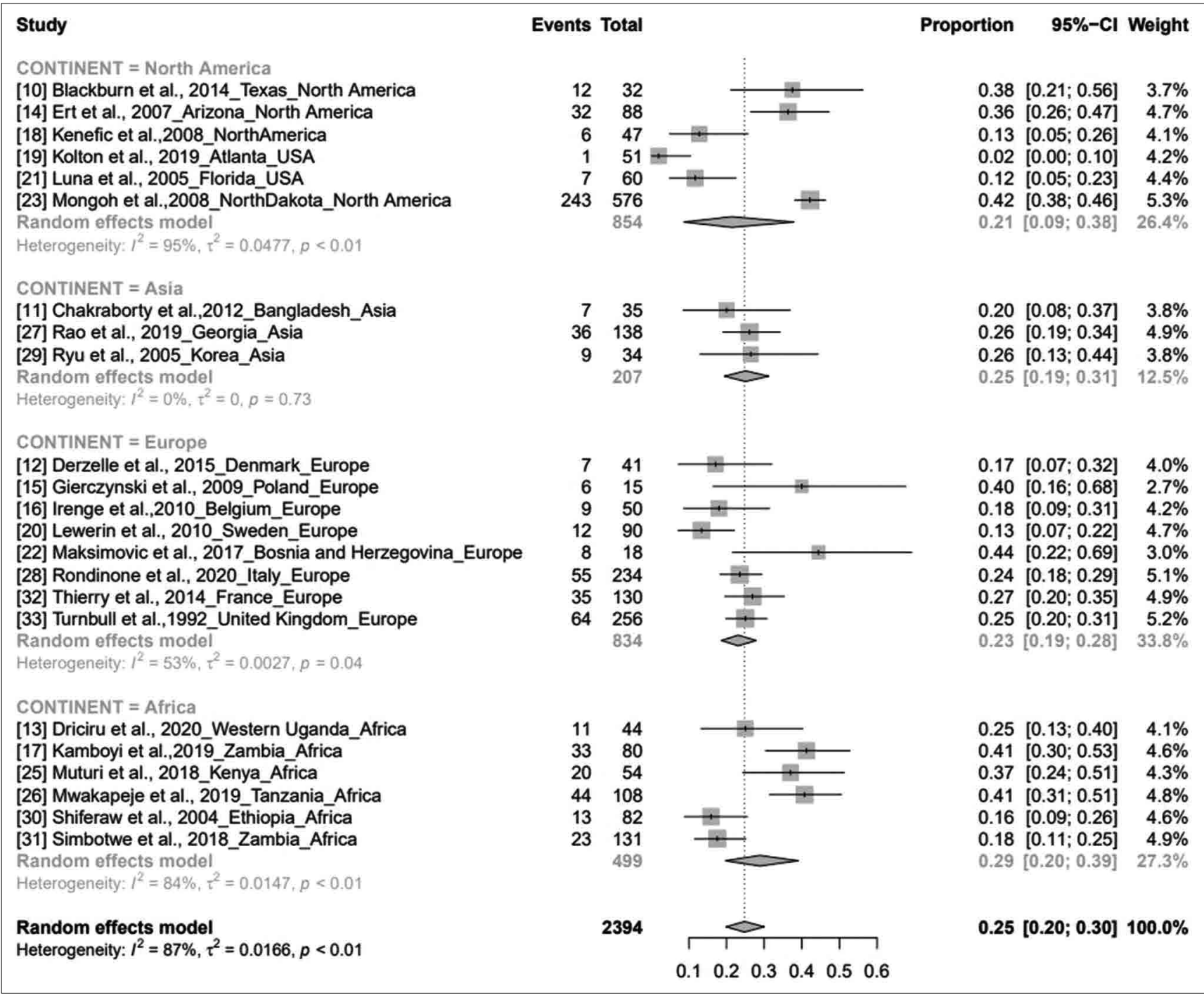

Figure-4: Forest plot showing anthrax prevalence in different continents. 


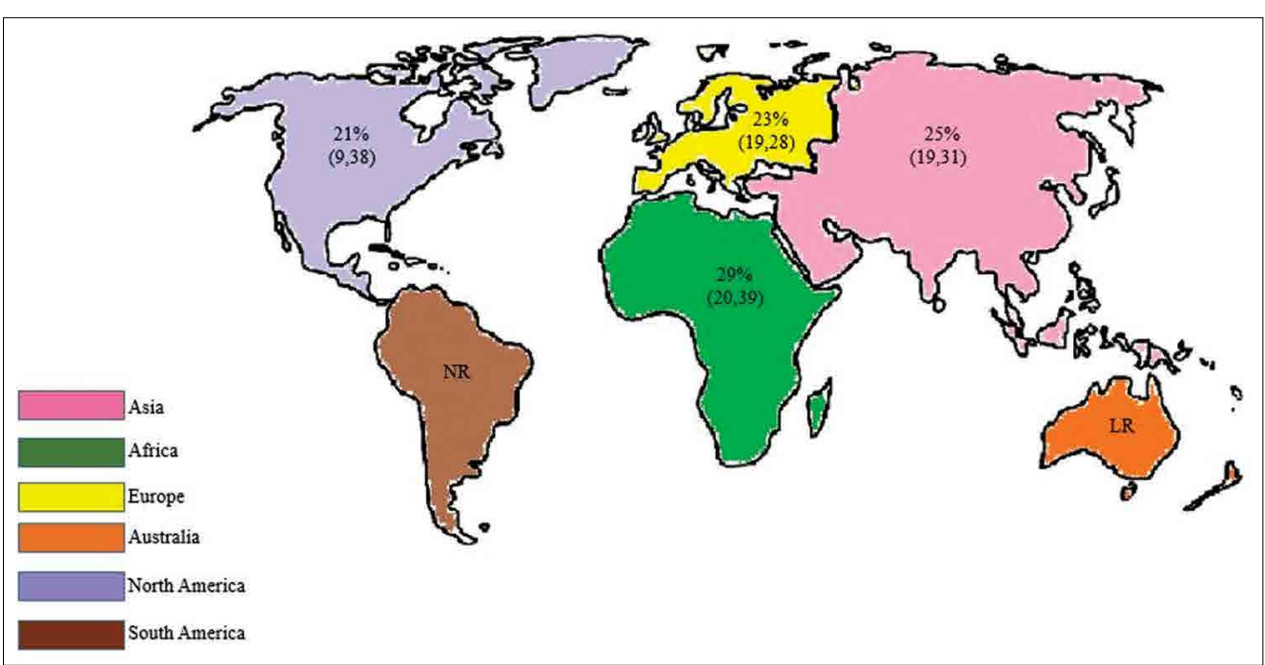

Figure-5: World map showing the continent-wise prevalence estimates of anthrax based on subgroup meta-analysis. NR: No reports, LR: Limited reports [Source; Raw map sourced from https://d-maps.com/index.php?lang=en].

decelerates the progress and sustainability of the livestock sector [34]. In general, anthrax outbreaks occur during the hot and humid transition period between the dry and wet seasons. During this period, soil temperatures are higher than usual in both day and night. The soil is also significantly disturbed during the cultivation season, which contributes to the multiplication of bacteria, increasing subsequent soil-borne infections of animals. Shiferaw et al. have considered Wabessa village from Dessie Zuria district in Ethiopia for the study [30]. The virulence of the $B$. anthracis strains is followed by two large plasmids, pXO1 and pXO2, and strains lacking either plasmid will become virulent or substantially weakened [35]. Disease spread in populations is a consequence of the interaction between host, pathogen, and environment, that is, the epidemiological chord. Yet, the effects of each chord component may vary dramatically in various settings [36]. Worldwide, domestic cattle are the most commonly reported livestock with anthrax [10]. The anthrax immunochromatographic test is considered the best diagnostic test for samples taken from animals suspected to have anthrax within $48 \mathrm{~h}$ of death, and this study showed a prevalence of $24 \%$ (95\% CI, 19-30\%) [11]. Standard single-nucleotide polymorphism (SNP) composing and entire genome sequencing were used to examine the subatomic variety of B. anthracis strains secluded from cows [12]. The Active Anthrax Detect Rapid Test parallel stream insusceptible test is a state-of-care test that was under investigational use for recognizing $B$. anthracis [19]. The earliest possible awareness of an anthrax attack could reduce illness and death. A progressing challenge to the observation approach is that no exact clinical calculation exists to openly recognize whether a bacterium is separated from blood culture or culture infection [37]. Although robust techniques to test the positivity of $B$. anthracis are lacking, certain studies have shown different methodologies for disease detection.

An ongoing PCR test was produced for the quick identification of $B$. anthracis [16,20,38]. Field arranged blood smears were verified by microscopy using four recoloring strategies, including PCR trailed by Bayesian latent class investigation [39]. Samples were tested using a 31-marker multilocus variable-number tandem repeat analysis (MLVA) to identify different genotypes. Different genotypes separated from the same animal could result either from freshly rising mutations during the incubation period of the disease or through mixed infections. The latter might feature grazing over several infectious sites or, otherwise, on one former carcass site carrying the different genotypes from the body fluids of the dead animal $[20,40]$. The test was sequentially attempted to detect $B$. anthracis through blood agar culture and Gram staining, which was then affirmed by multiplex PCR [17]. Tests were examined using MLVA and SNPs. Nonetheless, the examination of four single-nucleotide repeat markers decided these protect into six particular genotypes giving awareness on illness transmission $[18,41]$. Hereditary connections and atomic qualities of $34 \mathrm{~B}$. anthracis isolates from soil and medical samples in different areas are surveyed using the MLVA and amplified fragment length polymorphism approaches [29]. An indirect ELISA relative to recombinant protective antigen domain 1 of B. anthracis was created and used to identify anti-PA antibodies in cows [31]. This study provides insights into the review and analysis of publications on anthrax prevalence worldwide. According to the pooled literature, we did not find a meta-analysis on anthrax prevalence in livestock. Hence, we conducted this study.

This meta-analysis showed that the collective prevalence estimate from 1992 to 2020 was $24 \%$. It is observed from the pooled publications (Figure-2) that the global prevalence of anthrax varies between $2 \%$ and $44 \%$, and atmospheric temperature, soil characteristics, rainfall, and river floods contribute to the spread of the pathogen and have a profound effect on the disease $[17,42,43]$. However, the effects of precipitation or vegetative green-up on bacterial physiology 
or miniature nature to drive anthrax outbreaks are unknown [44]. The continent-wise pooled prevalence study showed that anthrax is most prevalent in Africa $(29 \%)$ and least prevalent in North America (21\%). As we could not find any publication from South America and only one publication from Australia related to this study, we excluded these continents in the subgroup analysis. The high prevalence of anthrax in West Uganda (Africa) was due to dry climatic conditions along with alkaline soils rich in calcium and potassium, hence leading to subsequent anthrax outbreaks, and this study showed a prevalence of $25 \%(95 \%$ CI, 13-40\%) [13]. In the study by Ert et al., anthrax prevalence was $36 \%$ (95\% CI, 26-47\%). The use of strain-specific SNPs for low-level and high-throughput genotyping can be an effective tool in real-world forensic and public health publications [14]. In Poland, the prevalence of $B$. anthracis is $40 \%(95 \%$ CI, 16-68\%), which is highly heterogeneous compared with those in other European countries [15]. Anthrax has emerged as a public health hazard in Zambia, with a prevalence of $48 \%$ (95\% CI, 25-64\%). Flooding, rainfall pattern, temperature, and evaporation along with epidemiological factors, such as cattle population, contributed to this high prevalence [17]. Disease incidence was $44 \%(95 \%$ CI, 22-69\%), which is directly proportional to climate change [22]. The highest prevalence in Africa may be because of the high livestock population and favorable climatic conditions prevalent in this region. The study showed a prevalence of $41 \%(95 \%$ CI, 31-51\%) [26]. Disease prevalence in Korea (Changnyeong Province) was $26 \%$ (95\% CI, 13-44\%]. Subsequently, the pooled study showed that the MLVA method may be significant for adaptation to environmental conditions of Asia [29]. Due to insufficient vaccination programs and the low percentage of seropositive cattle, a recurrent anthrax outbreak was noted in the Western Region of Zambia that showed a prevalence of $18 \%$ (95\% CI, 12-26\%) [31]. Anthrax is highly prevalent in West Africa, which is independent of the adequacy of animal immunizations in controlling anthrax, coordination, underreporting, and restricted assets, making actualizing vaccination campaigns difficult [45]. Anthrax is prevalent in Africa, the Middle East, some Asian countries, and South America. The disease has also been detected in Turkey and is mostly seen from April to November [46]. It is a neglected ailment, and its global dissemination remains under characterized. An estimated 1.8 billion individuals live within the anthrax suitable areas worldwide, an enormous number of whom live in rural areas in Africa, Europe, and Asia [47]. High-risk areas comprise 1.1 billion livestock (95\% CI: 404 million-2.3 billion), with 268.1 million cattle (95\% CI: 87.4-639 million), 320 million sheep (95\% CI: 138-622 million), 211.2 million goats (95\% CI: 74.8-453 million), 294.9 million pigs (95\% CI: $103-583$ million), and 0.6 million buffalo (95\% CI: 0.16-1.6 million) [46].
It is predictable from the overall pooled data that the prevalence of anthrax in livestock might continue to extend in the future unless we manage it effectively by adopting necessary arrangements.

As the meta-analysis included only published articles, it may have overestimated the actual effect level. The derived results are susceptible to the methodological quality of the articles included in the study.

\section{Conclusion}

This study estimated the pooled prevalence of anthrax worldwide in livestock using a systematic review and meta-analysis. As far as we could know, this is the first meta-analysis on the prevalence of anthrax in livestock from a global perspective. The prevalence rate of anthrax in Africa, Asia, and Europe was high, whereas that in North America was low. Hence, efficient early diagnostic strategies and scientific management practices should be implemented in high-risk regions with active involvement by veterinarians and information technologists to decrease the effects of infection and subsequently improve the economic status.

\section{Authors' Contributions}

BS: Collected the data, conducted the research work and wrote the manuscript. SS: Performed review and supervision. KPS: Guided the research steps. GL: Performed writing review and editing. SSP: Performed writing review and editing. GS: Performed visualization. All authors read and approved the final manuscript.

\section{Acknowledgments}

The authors are thankful to the Spatial Epidemiology Lab, Indian Council of Agricultural Research (ICAR) - National Institute of Veterinary Epidemiology and Disease Informatics (NIVEDI) Department of Veterinary Public Health and Epidemiology, Veterinary College and Outreach project on Zoonotic diseases, ICAR for providing necessary support to carry out this research work. The authors did not receive any specific funds for this study.

\section{Competing Interests}

The authors declare that they have no competing interests.

\section{Publisher's Note}

Veterinary World remains neutral with regard to jurisdictional claims in published map and institutional affiliation.

\section{References}

1. Dragon, D.C. and Rennie, R.P. (1995) The ecology of anthrax spores: Tough but not invincible. Can. Vet. J., 36(5): 295-301.

2. Barro, A.S., Fegan, M., Moloney, B., Porter, K., Muller, J., Warner, S. and Blackburn, J.K. (2016) Redefining the Australian anthrax belt: Modeling the ecological niche and predicting the geographic distribution of Bacillus anthracis. 
PLoS Negl. Trop. Dis., 10(6): e0004689.

3. Assefa, A., Bihon, A. and Tibebu, A. (2020) Anthrax in the Amhara regional state of Ethiopia; spatiotemporal analysis and environmental suitability modeling with an ensemble approach. Prev. Vet. Med., 184: 105155.

4. Paramanandham, K., Mohankumar, A., Suresh, K.P., Jacob, S.S. and Roy, P. (2019) Prevalence of Anaplasma species in India and the World in dairy animals: A systematic review and meta-analysis. Res. Vet. Sci., 123: 159-170.

5. Jain, V., Sharma, R. and Singh, S. (2012) Doing meta-analysis in research: A systematic approach. Indian J. Dermatol. Venereol. Leprol., 78(3): 242-250.

6. Jacob, S.S., Sengupta, P.R., Paramanandham, K., Suresh, K.P., Chamuah, J.K., Rudramurthy, G.R. and Roy, P. (2020) Bovine babesiosis: An insight into the global perspective on the disease distribution by systematic review and meta-analysis. Vet. Parasitol., 283: 109136.

7. Egger, M., Smith, G.D., Schneider, M. and Minder, C. (1997) Bias in meta-analysis detected by a simple, graphical test. BMJ, 315(7109): 629-634.

8. Higgins, J.P.T., Thompson, S.G., Deeks, J.J. and Altman, J.G. (2003) Measuring inconsistency in meta-analyses. $B M J, 327(7414)$ : 557-560.

9. Richardson, M, Garner, P. and Donegan, S. (2019) Interpretation of subgroup analyses in systematic reviews: A tutorial. Clinical Epidemiology and Global Health., 7(2): 192-198.

10. Blackburn, J.K., Van Ert, M., Mullins, J.C., Hadfield, T.L. and Hugh-Jones, M.E. (2014) The necrophagous fly anthrax transmission pathway: Empirical and genetic evidence from wildlife epizootics. Vector Borne Zoonotic Dis., 14(8): 576-583.

11. Chakraborty, A., Khan, S.U., Hasnat, M.A., Parveen, S., Islam, M.S., Mikolon, A., Chakraborty, R.K., Ahmed, B.N., Ara, K., Haider, N., Zaki, S.R., Hoffmaster, A.R., Rahman, M., Luby, S.P. and Hossain, M.J. (2012) Anthrax outbreaks in Bangladesh. Am. J. Trop. Med. Hyg., 86(4): 703-710.

12. Derzelle, S., Girault, G., Kokotovic, B. and Angen, O. (2015) Whole genome-sequencing and phylogenetic analysis of a historical collection of Bacillus anthracis strains from Danish Cattle. PLos One, 10(8): e0134699.

13. Driciru, M., Rwego, I.B., Ndimuligo, S.A., Travis, D.A., Mwakapeje, E.R., Craft, M., Asiimwe, B., Alvarez, J., Ayebare, S. and Pelican, K. (2020) Environmental determinants influencing anthrax distribution in Queen Elizabeth Protected Area, Western Uganda. PLos One, 15(8): e0237223.

14. Van Ert, M.N., Easterday, W.R., Simonson, T.S., Ren, J.M., Pearson, T., Kenefic, L.J., Busch, J.D., Huynh, L.Y., Dukerich, M., Trim, C.B., Beaudry, J., Welty-Bernard, A., Read, T., Fraser, C.M., Ravel, J. and Keim, P. (2007) Strainspecific single-nucleotide polymorphism assays for the Bacillus anthracis Ames strain. J. Clin. Microbiol., 45(1): 47-53.

15. Gierczynski, R., Jakubczak, A. and Jagielski, M. (2009) Extended multiple-locus variable-number tandem-repeat analysis of Bacillus anthracis strains isolated in Poland. Pol. J. Microbiol., 58(1): 3-7.

16. Irenge, L.M., Durant, J.F., Tomaso, H., Pilo, P., Olsen, J.S., Ramisse, V., Mahillon, J. and Gala, J.L. (2010) Development and validation of a real-time quantitative PCR assay for rapid identification of Bacillus anthracis in environmental samples. Appl. Microbiol. Biotechnol., 88(5): 1179-1192.

17. Kamboyi, H.K., de Garine-Wichatitsky, M., Hang'ombe, M.B. and Munyeme, M. (2019) Risk mapping and eco-anthropogenic assessment of anthrax in the upper Zambezi basin. Vet. Med. Sci., 5(3): 419-427.

18. Kenefic, L.J., Beaudry, J., Trim, C., Daly, R., Parmar, R., Zanecki, S., Huynh, L., Van Ert, M.N., Wagner, D.M., Graham, T. and Keim, P. (2007) High resolution genotyping of Bacillus anthracis outbreak strains using four highly mutable single nucleotide repeat markers. Lett. Appl.
Microbiol., 46(5): 600-603.

19. Kolton, C.B., Marston, C.K., Stoddard, R.A., Cossaboom, C., Salzer, J.S., Kozel, T.R., GatesHollingsworth, M.A., Cleveland, C.A., Thompson, A.T., Dalton, M.F., Yabsley, M.J. and Hoffmaster, A.R. (2019) Detection of Bacillus anthracis in animal tissues using In-Bios active anthrax detect rapid test lateral flow immunoassay. Lett. Appl. Microbiol., 68(6): 480-484.

20. Lewerin, S.S., Elvander, M., Westermark, T., Hartzell, L.N., Norström, A.K., Ehrs, S., Knutsson, R., Englund, S., Andersson, A.C., Granberg, M., Bäckman, S., Wikström, P. and Sandstedt, K. (2010) Anthrax outbreak in a Swedish beef cattle herd - 1st case in 27 years: Case report. Acta Vet. Scand., 52(1): 7.

21. Luna, V.A., Peak, K.K., Veguilla, W.O., Reeves, F., Heberlein-Larson, L., Cannons, A.C., Amuso, P. and Cattani, J. (2005) Use of two selective media and a broth motility test can aid in identification or exclusion of Bacillus anthracis. J. Clin. Microbiol., 43(9): 4336-4341.

22. Maksimovic, Z., Cornwell, M.S., Semren, O. and Rifatbegovic, M. (2017) The apparent role of climate change in a recent anthrax outbreak in cattle. Rev. Sci. Tech., 36(3): 959-963.

23. Mongoh, M.N., Dyer, N.W., Stoltenow, C.L. and Khaitsa, M.L. (2008) Risk factors associated with anthrax outbreak in animals in North Dakota, 2005: A retrospective case-control study. Public Health Rep., 123(3): 352-359.

24. Muller, J., Gwozdz, J., Hodgeman, R., Ainsworth, C., Kluver, P., Czarnecki, J., Warner, S. and Fegan, M. (2015) Diagnostic performance characteristics of a rapid field test for anthrax in cattle. Prev. Vet. Med., 120(3-4): 277-282.

25. Muturi, M., Gachohi, J., Mwatondo, A., Lekolool, I., Gakuya, F., Bett, A., Osoro, E., Bitek, A., Thumbi, S.M., Munyua, P., Oyas, H., Njagi, O.N., Bett, B. and Njenga, M.K. (2018) Recurrent Anthrax outbreaks in humans, livestock, and wildlife in the same locality, Kenya, 2014-2017. Am. J. Trop. Med. Hyg., 99(4): 833-839.

26. Mwakapeje, E.R., Ndimuligo, S.A., Mosomtai, G., Ayebare, S., Nyakarahuka, L., Nonga, H.E., Mdegela, R.H. and Skjerve, E. (2019) Ecological niche modeling as a tool for prediction of the potential geographic distribution of Bacillus anthracis spores in Tanzania. Int. J. Infect. Dis., 79: 142-151.

27. Rao, S., Traxler, R., Napetavaridze, T., Asanishvili, Z., Rukhadze, K., Maghlakelidze, G., Geleishvili, M., Broladze, M., Kokhreidze, M., Reynolds, D., Shadomy, S. and Salman, M. (2019) Risk factors associated with the occurrence of anthrax outbreaks in livestock in the country of Georgia: A case-control investigation 2013-2015. PLoS One, 14(5): e0215228.

28. Rondinone, V., Serrecchia, L., Parisi, A., Fasanella, A., Manzulli, V., Cipolletta, D. and Galante, D. (2020) Genetic characterization of Bacillus anthracis strains circulating in Italy from 1972 to 2018. PLoS One, 15(1): e0227875.

29. Ryu, C., Lee, K., Hawng, H.J., Yoo, C.K., Seong, W.K. and Oh, H.B. (2005) Molecular characterization of Korean Bacillus anthracis isolates by amplified fragment length polymorphism analysis and multilocus variable-number tandem repeat analysis. Appl. Environ. Microbiol., 71(8): 4664-4671

30. Shiferaw, G. (2004) Anthrax in Wabessa village in the Dessie Zuria district of Ethiopia. Rev. Sci. Tech., 23(3): 951-956.

31. Simbotwe, M., Fujikura, D., Ohnuma, M., Omori, R., Furuta, Y., Muuka, G.M., Hang'ombe, B.M. and Higashi, H. (2018) Development and application of a Bacillus anthracis protective antigen domain-1 in house ELISA for the detection of anti-protective antigen antibodies in cattle in Zambia, 2018. PLoS One, 13(10): e0205986.

32. Thierry, S., Tourtere, C., Le Fleche, P., Derzelle, S., Dekhil, N., Mendy, C., Colaneri, C., Vergnaud, G. and Madani, N. (2014) Genotyping of french Bacillus anthracis 
strains based on 31-loci multilocus VNTR analysis: Epidemiology, marker evaluation, and update of the internet genotype database. PLoS One, 9(6): e95131.

33. Turnbull, P.C., Doganay, M., Lindeyque, P.M., Aygen, B. and McLaughlin, J. (1992) Serology and anthrax in humans, livestock and Etosha National Park wildlife. Epidemiol. Infect., 108(2): 299-313.

34. Otlu, S., Sahin, M. and Genc, O. (2012) Occurrence of anthrax in Kars district, Turkey. Acta Vet. Hung, 50(1): 17-20.

35. Sushma, B., Seema, S., Suresh, K.P., Leena, G., Patil, S.S., Uma, B.I. and Chandan, S. (2021) Relative analysis of Codon Usage and Nucleotide Bias between Anthrax Toxin Genes Subsist Inpxo1 Plasmid of Bacillus anthracis. Ann. Romanian Soc. Cell Biol., 25(4): 5758 - 5774.

36. Kanankege, K.S.T., Abdrakhmanov, S.K., Alvarez, J., Glaser, L., Bender, J.B., Mukhanbetkaliyev, Y.Y., Korennoy, F.I., Kadyrov, A.S., Abdrakhmanova, A.S. and Perez, A.M. (2019) Comparison of spatiotemporal patterns of historic natural Anthrax outbreaks in Minnesota and Kazakhstan. PLoS One, 14(5): e0217144.

37. Begier, E.M., Barrett, N.L., Mshar, P.A., Johnson, D.G., Hadler, J.L. and Connecticut Bioterrorism Field Epidemiology Response Team. (2005) Gram-positive rod surveillance for early anthrax detection. Emerg. Infect. Dis., 11(9): 1483-1486.

38. Berg, T., Suddes, H., Morrice, G. and Hornitzky, M. (2006) Comparison of PCR, culture and microscopy of blood smears for the diagnosis of anthrax in sheep and cattle. Lett. Appl. Microbiol., 43(2): 181-186.

39. Aminu, O.R., Lembo, T., Zadoks, R.N., Biek, R., Lewis, S., Kiwelu, I., Mmbaga, B.T., Mshanga, D., Shirima, G., Denwood, M. and Forde, T.L. (2020) Practical and effective diagnosis of animal anthrax in endemic low-resource settings. PLoS One, 14(9): e0008655.
40. Beyer, W. and Turnbull, P.C.B. (2013) Co-infection of an animal with more than one genotype can occur in anthrax. Lett. Appl. Microbiol., 57(4): 380-384.

41. Rume, F.L., Affuso, A., Serrecchia, L., Rondinone, V., Manzulli, V., Campese, E., Di Taranto, P., Biswas, P.K., Ahsan, C.R., Yasmin, M., Fasanella, A. and Hugh-Jones, M. (2016) Genotype analysis of Bacillus anthracis strains circulating in Bangladesh. PLoS One, 11(4): e0153548.

42. Walsh, M.G., de Smalen, A.W. and Mor, S.M. (2018) Climatic influence on anthrax suitability in warming northern latitudes. Sci. Rep., 8(1): 9269.

43. Mullins, J., Lukhnova, L., Aikimbayev, A., Pazilov, Y., Van Ert, M. and Blackburn, J.K. (2011) Ecological niche modelling of the Bacillus anthracis A1. a sub-lineage in Kazakhstan. BMC Ecol., 11: 32.

44. Norris, M.H. and Blackburn, J.K. (2019) Linking geospatial and laboratory sciences to define mechanisms behind landscape level drivers of anthrax outbreaks. Int. J. Environ. Res. Public Health, 16(19): 3747.

45. Kracalik, I.T., Kenu, E., Ayamdooh, E.N., AllegyeCudjoe, E., Polkuu, P.N., Frimpong, J.A., Nyarko, K.M., Bower, W.A., Traxler, R. and Blackburn, J.K. (2017) Modeling the environmental suitability of anthrax in Ghana and estimating populations at risk: Implications for vaccination and control. PLoS Negl. Trop. Dis., 11(10): e0005885.

46. Ozkurt, Z., Parlak, M., Tastan, R., Dinler, U., Saglam, Y.S. and Ozyurek, S.F. (2005) Anthrax in Eastern Turkey, 19922004. Emerg. Infect. Dis., 11(12): 1939-1941.

47. Carlson, C.J., Kracalik, I.T., Ross, N., Alexander, K.A., Hugh-Jones, M.E., Fegan, M., Elkin, B.T., Epp, T., Shury, T.K., Zhang, W., Bagirova, M., Getz, W.M. and Blackburn, J.K. (2019) The global distribution of Bacillus anthracis and associated anthrax risk to humans, livestock and wildlife. Nat. Microbiol., 4(8): 1337-1343. 